\title{
Influence of pneumococcal serotype group on outcome in adults with bacteraemic pneumonia
}

\author{
M. Luján*,\#, M. Gallego*,\#, Y. Belmonte*, D. Fontanals ${ }^{\Uparrow}$, J. Vallès ${ }^{\#,+}$,

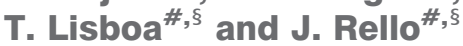

ABSTRACT: The influence of infecting serotype group on outcome in bacteraemic pneumococcal pneumonia remains unclear.

We performed a prospective, 10-yr observational study in an 800-bed teaching hospital. 299 adults diagnosed with pneumonia whose blood cultures showed growth of Streptococcus pneumoniae were included in the study. High invasive disease potential $(H)$ serotypes included serotypes 1, 5 and 7F, which served as a reference category, were compared with low invasive disease potential (L) serotypes (3, 6A, 6B, 8, 19F, and 23F) and other (O) serotypes (non-H, non-L). The influence on outcome was determined for each group of serotypes after adjusting for underlying conditions and severity of illness at admission.

Overall, 30-day mortality was $11 \%$. H serotypes $(n=93)$ infected primarily younger people and presented a higher risk of complicated parapneumonic effusion or empyema (17.2 versus $5.1 \%$; $p=0.01)$, with lower mortality $(3.2 \%)$. The isolation of $L$ serotypes $(n=78)$ was an independent risk factor for 30-day mortality (OR 7.02, 95\% Cl 1.72-28.61), as were Charlson score (OR 1.30, 95\% Cl 1.08-1.58), alcohol abuse (OR 3.99, 95\% Cl 1.39-11.39) and severity of illness measured by American Thoracic Society (ATS)/Infectious Diseases Society of America (IDSA) criteria (OR 4.80, 95\% Cl 1.89-12.13).

A vaccination strategy including serotypes $3,6 \mathrm{~A}, 6 \mathrm{~B}, 8,19 \mathrm{~F}$ and $23 \mathrm{~F}$ may improve survival in adults.

KEYWORDS: Bacteraemic pneumococcal pneumonia, conjugated vaccines, serotypes, Streptococcus pneumoniae

acteraemic pneumococcal pneumonia accounts for $9-18$ cases per 100,000 adults [1]. The outcome is a complex process that depends on interactions between factors related to the host [2,3], therapy [4-6] and microorganism [7, 8], including the bacterial pneumococcal load [9].

The polysaccharide capsule is considered to be the primary virulence factor of the pneumococcus, protecting it against phagocytosis. The classification of pneumococci into serotypes is based on the differences in the structure of the capsule and, to date, 90 different serotypes have been described [10]. Individual serotypes appear to have different clinical manifestations, but the data available to clarify whether different serotypes are associated with differing mortality rates are limited and sometimes contradictory.

In the paediatric population, BRUEGGEMAN et al. [11] described the invasive disease potential of several serotypes and the case-fatality rates for each group. According to their findings, a group of serotypes including 1, 5 and $7 \mathrm{~F}$ (known as invasive serotypes in the literature) rarely cause oropharyngeal colonisation but commonly cause bacteraemia (with a lower case-fatality rate). A second group of serotypes $(3,6 \mathrm{~A}, 6 \mathrm{~B}, 8,19 \mathrm{~F}$ and 23F) are frequently associated with colonisation but rarely cause bacteraemia, acting as opportunistic pathogens or microorganisms with low invasive disease potential. Higher case-fatality rates have been reported in children infected by these serotypes. In adults, the influence of serotypes on mortality remains controversial: some studies [12] found higher case-fatality rates for certain serotypes whereas others did not [13]. These results deserve attention in an era of the design and licensing of new formulations of pneumococcal conjugate vaccines (PCV).

The objective of our study was to determine the influence of infecting serotypes on the severity of
AFFILIATIONS

*Pneumology Service,

"Laboratory Service, UDIAT,

+Critical Care Service, Corporació

Parc Tauli, Departament de Medicina Universitat Autònoma de Barcelona, Sabadell,

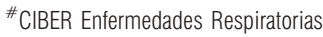
(CIBERes), Fundación CaubetCimera, Recinto Hospital Joan March, Bunyola, and

${ }^{\S}$ Critical Care Dept, Joan XXIII University Hospital, Rovira i Virgili University-IISPV, Tarragona, Spain.

CORRESPONDENCE

M. Luján

Pulmonary Medicine, Corporació

Parc Taulí

Hospital de Sabadell

Parc Taulí s/n

Sabadell

Barcelona 08208

Spain

E-mail: mlujan@tauli.cat

Received:

Nov 062009

Accepted after revision:

Jan 272010

First published online:

Feb 112010 
clinical presentation and outcome in a cohort of adults with bacteraemic pneumococcal pneumonia, after adjusting for underlying disease and severity of illness at admission. Our hypothesis was that the outcome for specific groups of serotypes would be different when adjusted for baseline conditions of the host and severity of illness at admission.

\section{METHODS}

\section{Study population}

This study was performed between January 1999 and March 2009 in an 800-bed teaching hospital. All patients included in the study were aged $\geqslant 18$ yrs, had a diagnosis of pneumonia (fever, productive cough, chest pain, shortness of breath and crackles on auscultation in addition to a chest radiograph interpreted as pneumonia) and their blood cultures, obtained within the first $48 \mathrm{~h}$ of hospitalisation showed growth of Streptococcus pneumoniae. Exclusion criteria were bacteraemia

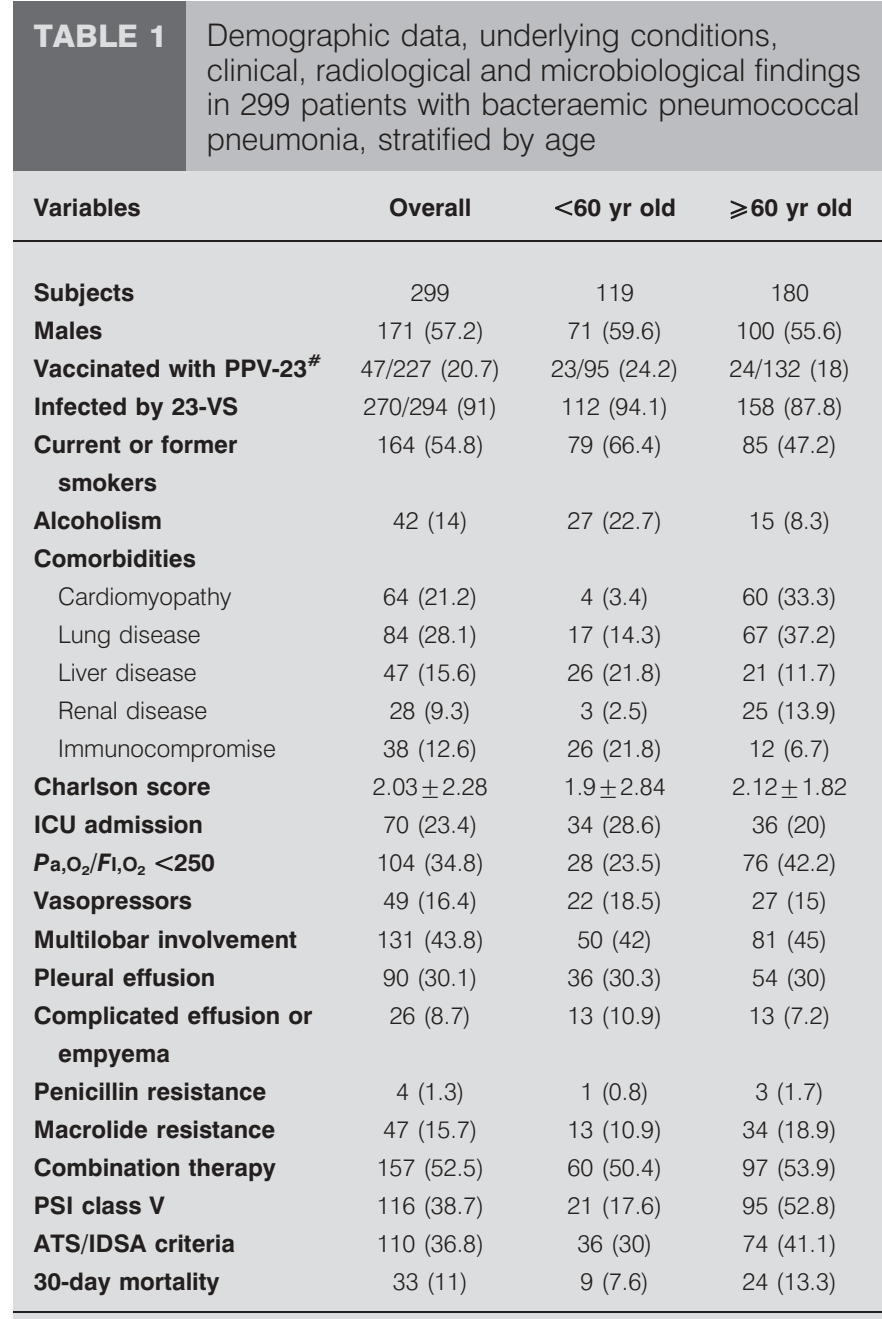

Data are presented as $n, n(\%)$ or mean \pm SD. PPV-23: 23-valent pneumococcal polysaccharide vaccine; 23-VS: PPV-23 vaccine serotypes; ICU: intensive care unit; $\mathrm{Pa}, \mathrm{O}_{2}$ : arterial oxygen tension; $\mathrm{Fl}, \mathrm{O}_{2}$ : inspiratory oxygen fraction; PSI: Pneumonia Severity Index; ATS: American Thoracic Society; IDSA: Infectious Diseases Society of America. ${ }^{*}$ : patients vaccinated within the last 5 yrs among the 194 who fulfilled vaccination criteria. from other sources, the presence of concurrent meningitis or endocarditis at admission, and growth of other pathogenic microorganisms in the blood cultures. The study was approved by the institutional research board and informed consent was waived.

The following variables were recorded at admission: age, sex, tobacco and alcohol abuse, underlying diseases, vaccination status with the 23-valent polysaccharide (PPV-23) vaccine (patients who received the last administration of the vaccine in the 5 yrs before pneumonia were considered as vaccinated), symptoms in the days prior to and at admission, relevant physical findings, blood gases, white and red blood cell count, blood urea nitrogen, serum creatinine, and chest radiograph pattern. The Charlson Score and Pneumonia Severity Index (PSI) score were calculated as reported elsewhere [14, 15]. Severity of pneumonia was assessed according to the prediction rule of the Infectious Diseases Society of America (IDSA)/ American Thoracic Society (ATS) considering severe pneumonia those cases that met at least 1 of 2 major criteria or 3 of 9 minor criteria [1].

During hospitalisation, the following variables were also recorded: in vitro susceptibility for strains, need for intensive care unit admission, vasoactive drugs and mechanical ventilation, major complications (respiratory failure, septic shock, empyema), outcome (30-day mortality), length of stay for survivors, and antibiotic therapy prescribed. Antibiotic therapy was not standardised and was left to the discretion of the attending physician.

\section{Definitions}

We defined the following as underlying conditions: alcohol consumption $\left(>60 \mathrm{~g} \cdot \mathrm{day}^{-1}\right)$, history of lung comorbidity (chronic obstructive pulmonary disease and interstitial lung disease), cardiac comorbidity (diagnosis of congestive heart failure, coronary artery disease or advanced valvulopathy), renal disease (chronic renal failure, with creatinine levels $\geqslant 1.5 \mathrm{mg} \cdot \mathrm{dL}^{-1}$ ), liver disease (biopsy-proven cirrhosis or diagnosis of viral or toxic chronic liver disease), diabetes mellitus (treatment with oral hypoglycaemics or insulin) and immunocompromise (corticosteroid therapy with $4 \mathrm{mg}$ prednisolone per day or equivalent for $>1$ month, HIV infection, haematological disease with immune defects or recent therapy with other immunosuppressors, including antineoplastic agents).

Antibiotic treatment was considered concordant if at least one of the antibiotics administered during the first $8 \mathrm{~h}$ showed full in vitro susceptibility in the isolated strain [5]. Complicated pleural effusion and empyema were defined according to current criteria [16].

\section{Microbiological evaluation}

Blood samples were processed using the BacT-Alert ${ }_{\circledast}$ system (bioMerieux, Lyon, France). Identification was carried out by colonial morphology on blood agar, Gram stain, susceptibility to optochin and detection of the colony antigen. Minimum inhibitory concentration (MIC) values were determined by the microdilution method in cation-adjusted Mueller-Hinton broth, supplemented with 5\% lysed horse blood. Penicillin and erythromycin susceptibility was assessed post hoc, converting MIC values to categories of susceptibility according to 


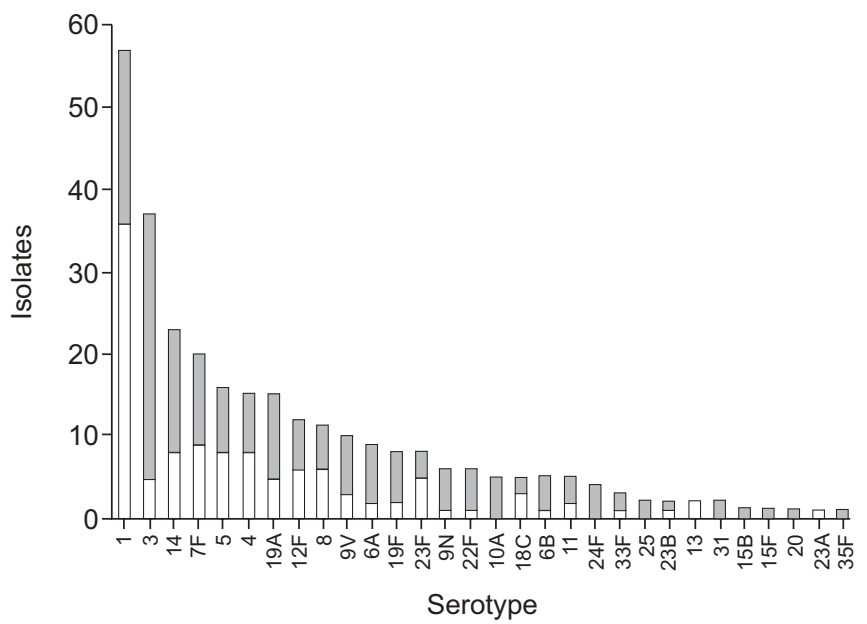

FIGURE 1. Distribution of serotypes among patients aged $\geqslant 60(\square)$ or $<60$ yrs old $(\square) . n=294$.

the 2008 Clinical and Laboratoru Standards Institute (CLSI) breakpoints [17]. All strains were sent to the Pneumococci Reference Laboratory (Instituto de Salud Carlos III, Majadahonda, Spain) for verification of sensitivity to the antibiotics and serotyping using the Quellung reaction and/ or dot blot assay, with the use of antisera provided by the Statens Serum Institute (Copenhagen, Denmark).

Serotypes were analysed individually and classified as follows. 1) Serotypes with high invasive disease potential (H group) or invasive serotypes [11]: 1, 5 and 7 F. 2) Low invasive potential (L group) or opportunistic serotypes: 3, 6A, 6B, 8, 19F and 23F [11]. 3) Serotypes not included in the $H$ and $L$ groups were denominated as other serotypes (O group).

For analysis of the impact of PPV-23, serotypes were classified as vaccine serotypes (included in PPV-23) and non-vaccine serotypes.

\section{Statistical analyses}

For quantitative variables (age and Charlson score), mean \pm SD were compared in the three groups of serotypes by means of an ANOVA test with Bonferroni's post hoc analysis. Dichotomous variables were evaluated using the Chi-squared test and Fisher's test when appropriate. Variables with $p<0.05$ (two-tailed) were considered significant. Severity according to PSI class and serotype group was assessed by logistic ordinal regression, $\mathrm{H}$ serotypes being the reference category. Survival was analysed with the Kaplan-Meier method and curves for groups of serotypes were compared by log-rank analysis. A multivariate logistic regression analysis was performed, considering variables with $\mathrm{p}<0.1$ in the univariate analysis. We restricted the number of variables included in the multivariate model following the rule of at least five to seven events (deaths) per variable [18]. Thus, variables showing colinearity (ATS/IDSA or PSI scores and individual criteria contained in them, individual comorbidities, and Charlson score) were not included together in the model. The appropriateness of the model was assessed by Hosmer-Lemeshow goodness-of-fit. Data were analysed using the SPSS-17 statistical package (SPSS Inc, Chicago, IL, USA).

\section{RESULTS}

299 consecutive adults were included in the study. Mean \pm SD age was 62.2 \pm 19.5 yrs (range 18-92 yrs). Overall, 30-day mortality was $11 \%$ (33 out of 299). Details on comorbidities and the main clinical, radiological and laboratory findings are shown in table 1 . The most frequently prescribed antibiotic therapies were combination therapies, including ceftriaxone or cefotaxime plus a macrolide $(44.4 \%)$, amoxicillin-clavulanate $(20 \%)$, ceftriaxone or cefotaxime $(11.7 \%)$, and levofloxacin $(11.6 \%)$ in monotherapy. Combination therapy was prescribed in $51 \%$ of patients who survived and in $67 \%$ of patients who died $(\mathrm{p}=0.08)$

Using the 2008 CLSI breakpoints, nonsusceptibility to penicillin (MIC $\geqslant 4 \mathrm{mg} \cdot \mathrm{mL}^{-1}$ ) was documented in only four $(1.3 \%)$ episodes and resistance to macrolides in 47 (15.7\%). Only four patients received discordant empirical therapy (one died; 25 versus $11 \%$; $\mathrm{p}=0.37$ ).

The infecting serotype was determined in 294 out of 299 patients. Figure 1 shows the distribution of serotypes between two age groups ( $<60$ and $\geqslant 60 \mathrm{yrs}$ old). Serotypes 1 (18.9\%), 3 $(12.3 \%)$ and $14(7.6 \%)$ were the most prevalent in the cohort. L serotypes infected mainly people aged $\geqslant 60$ yrs old (32 versus $17.8 \%$; OR 3.92, 95\% CI 2.05-7.51; $<<0.05$ ) Serotypes in patients who died are listed in table 2.

Table 3 compares underlying conditions, severity of illness on clinical presentation, antibiotic susceptibility and outcome for each group of serotypes. Patients with pneumonia caused by group $\mathrm{H}$ serotypes were significantly younger, with better baseline status (lower Charlson score) and presented a significantly higher incidence of complicated parapneumonic effusion or empyema ( $50 \%$ of these complicated effusions were associated with serotype 1).

\begin{tabular}{lcc} 
TABLE 2 & $\begin{array}{l}\text { Serotypes in patients who died }(\mathrm{n}=33) \text { within } 30 \\
\text { days of diagnosis of bacteraemic pneumococcal } \\
\text { pneumonia }\end{array}$ \\
Serotype & Isolates & Deaths \\
\hline $\mathbf{3}$ & $37(12.5)$ & 8 \\
$\mathbf{1 9 F}$ & $8(2.6)$ & 6 \\
$\mathbf{2 3 F}$ & $8(2.6)$ & 3 \\
$\mathbf{5}$ & $16(5.3)$ & 2 \\
$\mathbf{4}$ & $15(4.9)$ & 2 \\
$\mathbf{6 B}$ & $5(1.6)$ & 2 \\
$\mathbf{1 4}$ & $23(7.6)$ & 1 \\
$\mathbf{7 F}$ & $20(6.6)$ & 1 \\
$\mathbf{1 9 A}$ & $15(4.9)$ & 1 \\
$\mathbf{8}$ & $11(3.6)$ & 1 \\
$\mathbf{9 V}$ & $10(3.3)$ & 1 \\
$\mathbf{1 1 A}$ & $5(1.6)$ & 1 \\
$\mathbf{1 2 F}$ & $5(1.6)$ & 1 \\
$\mathbf{2 4}$ & $4(1.3)$ & 1 \\
$\mathbf{1 6 F}$ & $1(0.3)$ & 1 \\
$\mathbf{U n k n o w n}$ & $5(1.6)$ &
\end{tabular}

Data are presented as $n(\%)$ or $n$. 


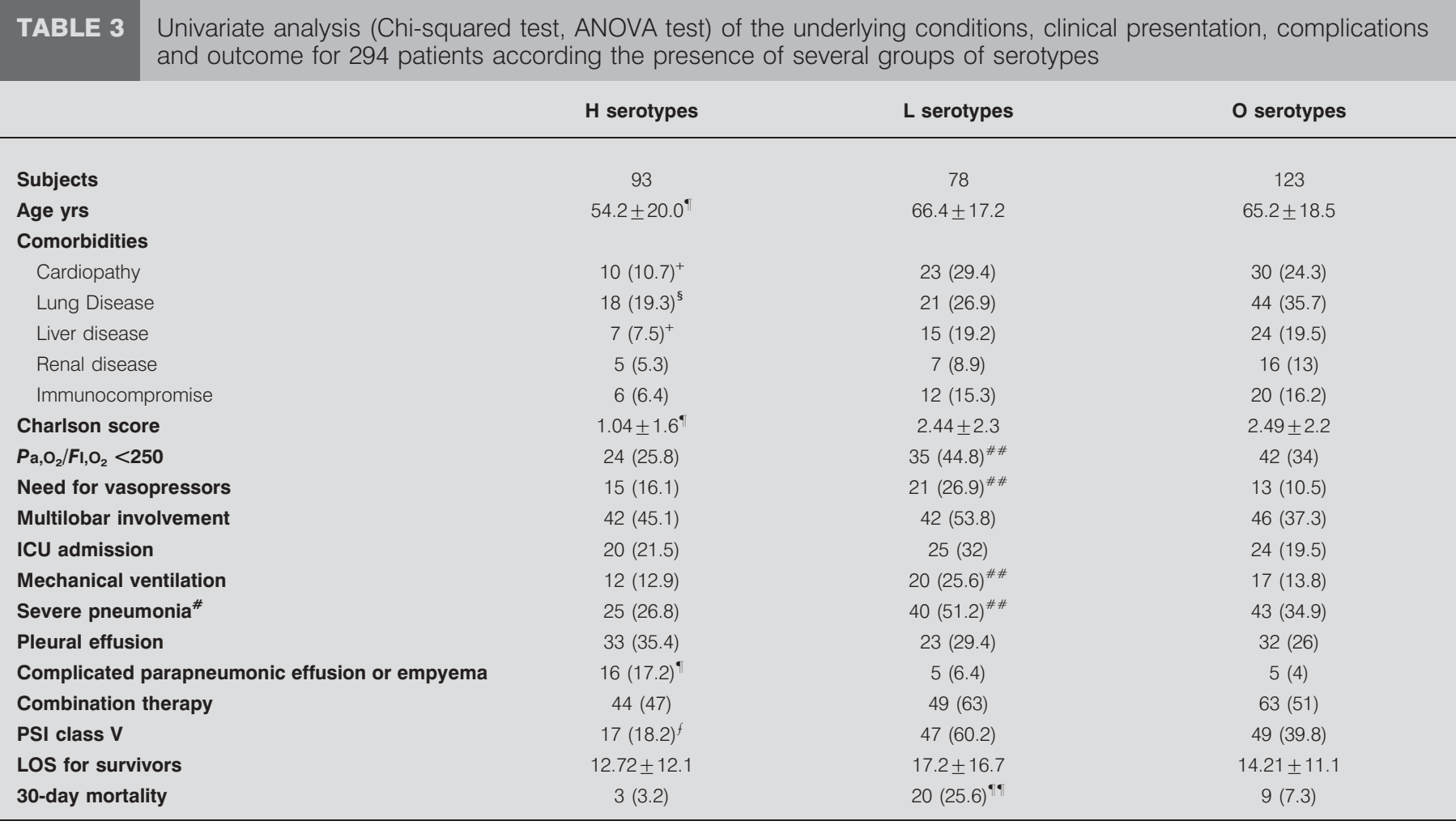

Data are presented as $n$, mean \pm SD or $n(\%)$, unless otherwise stated. $\mathrm{H}$ serotypes: 1,5 and $7 \mathrm{~F}$. L serotypes: 3, 6A, 6B, 8, 19F and 23F. O: Other serotypes. Pa, $\mathrm{O}_{2}$ : arterial oxygen tension; $\mathrm{Fl}, \mathrm{O}_{2}$ : inspiratory oxygen fraction; ICU: intensive care unit; PSI: Pneumonia Severity Index; LOS: length of stay. * : Pneumonia was considered severe when patients met at least one of two major American Thoracic Society (ATS)/Infectious Diseases Society of America (IDSA) criteria or three of the nine minor ATS/IDSA criteria [1]. ": $\mathrm{p}<0.001$ between group $\mathrm{H}$ and the other two groups. ${ }^{+}$: $\mathrm{p}<0.05$ between group $\mathrm{H}$ and the other two groups (Chi-squared test). ${ }^{\text {s: }} \mathrm{p}<0.05$ between groups $\mathrm{H}$ and $\mathrm{O} .{ }^{f}: \mathrm{p}<0.05$ between three groups. ${ }^{\# \#:} \mathrm{p}<0.05$ between group $\mathrm{L}$ and the other two groups. ${ }^{~}{ }: p<0.001$ between group $\mathrm{L}$ and the other two groups.

Although we did not find statistically significant differences in age or baseline conditions between groups $\mathrm{L}$ and $\mathrm{O}$, patients with pneumonia due to L serotypes not only presented higher mortality but also had severe pneumonia (ATS/IDSA criteria and PSI class V) more frequently, were more likely to have a arterial oxygen tension $\left(\mathrm{Pa}_{\mathrm{a}} \mathrm{O}_{2}\right) /$ inspiratory oxygen fraction $\left(\mathrm{FI}, \mathrm{O}_{2}\right)$ ratio $<250$ at admission, and had a greater need for vasopressors and mechanical ventilation (table 3). Moreover, differences in ATS/IDSA criteria were found both in major $(31 \%$ in $\mathrm{L}, 17.2 \%$ in $\mathrm{H}$ and $18 \%$ in $\mathrm{O}$ group; $\mathrm{p}<0.05)$ and minor criteria (51.2\% in L group, $23.6 \%$ in $\mathrm{H}$ and $32.5 \%$ for $\mathrm{O}$ group; $\mathrm{p}<0.01$ ). Figure 2 reflects the proportion of PSI class for each group of serotypes. Both $\mathrm{O}$ and $\mathrm{L}$ serotype isolates presented an increased likelihood of being associated with higher PSI classes when compared with $\mathrm{H}$ group (ordinal OR 3.25, 95\% CI 1.97-5.37 $(p<0.001)$ for O serotypes and ordinal OR 5.86, 95\% CI 3.26-10.53 ( $\mathrm{p}<0.001)$ for L serotypes). At the same time, an increased likelihood was also documented when $\mathrm{L}$ were compared with O serotypes (ordinal OR 1.97, 95\% CI 1.043.09; $\mathrm{p}<0.05)$.

Univariate analysis revealed that 30-day mortality was influenced by several underlying conditions, such as Charlson score and alcohol consumption, and by others related to severity of the clinical presentation (level of consciousness, need for vasopressors, multilobar involvement, $\mathrm{Pa}_{1} \mathrm{O}_{2} / \mathrm{FI}, \mathrm{O}_{2}$ ratio) and infecting serotype (group L) (table 4). Kaplan-Meier curves of patients infected by L serotypes versus those infected with $\mathrm{O}$ and $\mathrm{H}$ serotypes are shown in figure 3; there were statistically significant differences in survival (log-rank 26.55; p-value

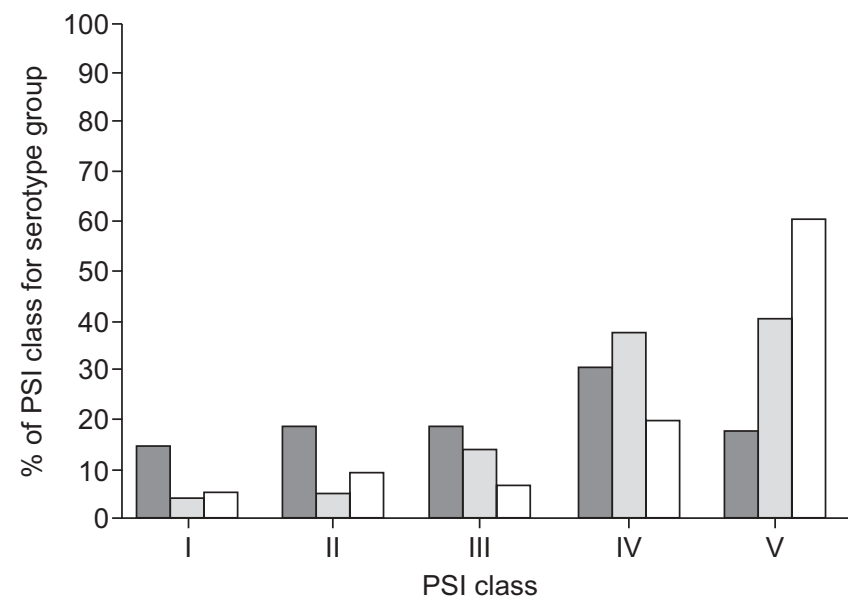

FIGURE 2. Distribution of Pneumonia Severity Index (PSI) classes in each serotype group. Both $\mathrm{O}(\square)$ and $L(\square)$ serotype isolates presented an increased likelihood of association with higher PSI classes (logistic ordinal regression). $\mathrm{H}$ serotypes ( $\square$ ) include serotypes 1,5 and $7 F$; L serotypes include serotypes 3, 6A, 6B, 8, 19F and 23F; O serotypes include non-H, non-L serotypes. 


\begin{tabular}{|c|c|c|c|}
\hline \multirow[t]{2}{*}{ TABLE 4} & \multicolumn{3}{|c|}{$\begin{array}{l}\text { Univariate model (Chi-squared test) for 30-day } \\
\text { mortality, including potential risk factors } \\
\text { influencing outcome related to underlying } \\
\text { conditions, severity of the clinical presentation, } \\
\text { and microbiological and therapy-related factors }\end{array}$} \\
\hline & & OR (95\% Cl) & p-value \\
\hline \multicolumn{4}{|c|}{ Underlying and demographic conditions } \\
\hline Male sex & & $4.81(1.80-12.55)$ & $<0.01$ \\
\hline Age & & $1.02(0.99-1.04)$ & 0.08 \\
\hline Alcoholism & & $3.79(1.67-8.56)$ & $<0.01$ \\
\hline Tobacco co & sumption & $2.03(0.94-4.45)$ & 0.07 \\
\hline Charlson sc & & $1.25(1.09-1.44)$ & $<0.01$ \\
\hline Immunocom & bromise & $2.51(1.04-6.08)$ & 0.05 \\
\hline \multicolumn{4}{|c|}{ Severity at admission } \\
\hline $\mathrm{Pa}, \mathrm{O}_{2} / \mathrm{Fl}_{1} \mathrm{O}_{2}<$ & & $6.20(2.75-13.93)$ & $<0.001$ \\
\hline Multilobar in & olvement & $5.59(2.38-13.59)$ & $<0.001$ \\
\hline Need for va & opressors & $4.15(1.90-9-07)$ & $<0.001$ \\
\hline Need for me & chanical ventilation & $3.53(1.70-7.79)$ & 0.01 \\
\hline \multicolumn{4}{|c|}{ Severity of illness scores } \\
\hline PSI class $V$ & & $14.91(5.08-43.9)$ & $<0.001$ \\
\hline ATS/IDSA cr & eria & $6.65(2.88-15.36)$ & $<0.001$ \\
\hline \multicolumn{4}{|c|}{ Antibiotic resistance } \\
\hline \multicolumn{4}{|c|}{ Penicillin } \\
\hline Macrolide & & $4.43(2.02-9.72)$ & $<0.01$ \\
\hline \multicolumn{4}{|l|}{ Microbiology } \\
\hline H serotypes & & 1 & \\
\hline O serotypes & & $2.36(0.63-9.05)$ & 0.2 \\
\hline L serotypes & & $10.34(2.94-36.5)$ & $<0.001$ \\
\hline \multicolumn{4}{|c|}{ Antibiotic therapy } \\
\hline Monotherap & & $1.94(0.90-4.16)$ & 0.1 \\
\hline Combinatior & therapy & 1 & \\
\hline
\end{tabular}

H serotypes (reference category): 1,5 and 7F. L serotypes: 3, 6A, 6B, 8, 19F and $23 \mathrm{~F}$. $\mathrm{O}$ serotypes: non- $\mathrm{L}$, non- $\mathrm{H}$ serotypes. $\mathrm{Pa}_{\mathrm{O}_{2}}$ : arterial oxygen tension; $\mathrm{Fl}, \mathrm{O}_{2}$ : inspiratory oxygen fraction; PSI: Pneumonia Severity Index; ATS: American Thoracic Society; IDSA: Infectious Diseases Society of America.

$<0.001)$. Finally, details of a logistic regression analysis after adjusting for baseline conditions and severity of illness that reached statistical significance in the univariate analysis are summarized in table 5. Two different models were analysed, with ATS/IDSA criteria in model A (Hosmer-Lemeshow goodness-of-fit 0.712) and PSI in model B (Hosmer-Lemeshow goodness-of-fit 0.682).

\section{DISCUSSION}

In our cohort, serotypes 3, 6A, 6B, 8, 19F and 23F (L serotypes) were involved in only $26 \%$ of adult bacteraemic pneumococcal pneumonia, but in two-thirds of fatal episodes. The L serotype group emerged as an independent variable of death, with a seven-fold increase compared with $\mathrm{H}$ serotypes and a four-fold increase compared with $\mathrm{O}$ serotypes. Moreover, patients infected with L serotypes also presented more hypoxaemia and higher need for vasopressors and mechanical ventilation, and were most likely to develop severe pneumonia (ATS/IDSA criteria and PSI class V). For their part, H serotypes (1, 5 and $7 F)$

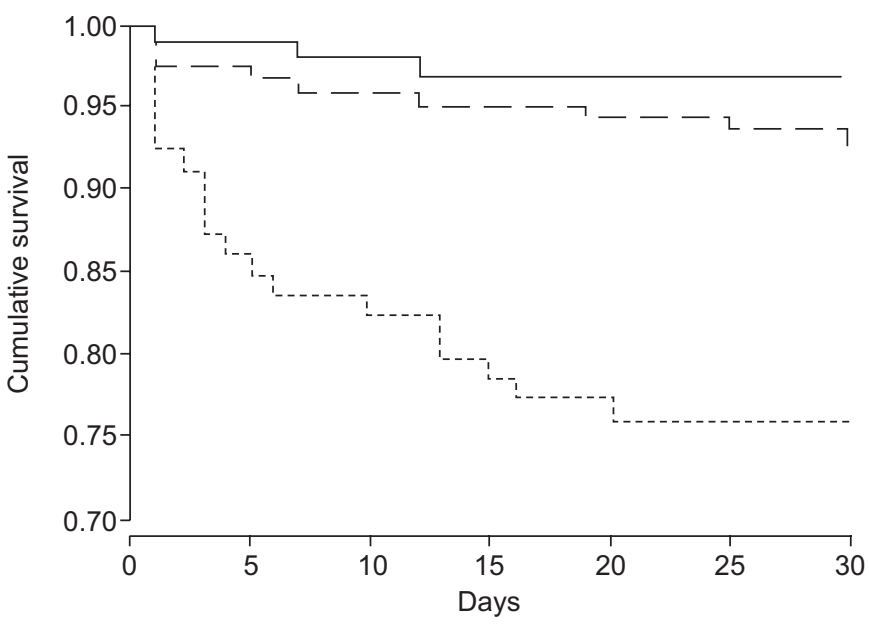

FIGURE 3. Kaplan-Meier survival curve for three groups of serotypes (censored at 30 days). $H$ serotypes $(-): 1,5$ and $7 \mathrm{~F}$; $L$ serotypes (---): 3, 6A, $6 \mathrm{~B}, 8,19 \mathrm{~F}$ and $23 \mathrm{~F}$; O serotypes $(---)$ : non- $\mathrm{H}$, non- $\mathrm{L}$ serotypes. $\mathrm{p}<0.001$ by log rank test.

infected younger people and presented a higher incidence of complicated parapneumonic effusion or empyema.

The excess of mortality attributable to certain pneumococcal serotypes in pneumococcal invasive disease has been a subject of controversy. In an unadjusted model, HENRIQUES et al. [19] found increased case-fatality rates for serotypes 3,6B and 19F. Using the same classification as in our study, SJOSTROM et al. [12] reported that invasive serotypes (1, 5 and 7F) were associated with lower mortality. Less invasive serotypes (3, 6A, $6 \mathrm{~B}, 8,19 \mathrm{~F}$ and $23 \mathrm{~F}$ ) infected patients with comorbidity and presented an increased case-fatality rate. In a retrospective study of 464 patients with pneumococcal invasive disease, MARTENS et al. [20] found that serotype 3 was linked to higher mortality in the global cohort (OR 2.63), but only found a trend in patients with pneumonia $(\mathrm{p}=0.06)$.

Differences in the clinical behaviour of serotypes are supported by a population-based study involving $>18,000$ patients with invasive pneumococcal disease. HARBOE et al. [21] reported different odds ratios for each serotype after adjusting for age, sex, meningitis and comorbidity level. Interestingly, not only the mortality but also the probability of infection by specific serotypes varied among different levels of comorbidity measured by the Charlson score. Moreover, infection by serotypes 3 , $19 \mathrm{~F}$ and $23 \mathrm{~F}$ was independently associated with mortality in patients with a high comorbidity level. Unfortunately, the authors did not present data for serotype distribution between different age groups among their adult population. As shown in figure 1, in our study, the distribution between patients aged $\geqslant 60$ or $<60$ yrs old was clearly different.

A recent retrospective study found that serotypes with low invasive disease potential presented higher prevalence of meningitis and primary bacteraemia, and higher case-fatality rate [22], but it remains unclear whether this mortality was associated with the infecting serotypes or with the source of the bacteraemia. In contrast, our study included only bacteraemic pneumonia: patients with meningitis or bacteraemia of unknown source at admission were excluded, demonstrating 


\begin{tabular}{|c|c|c|c|c|}
\hline \multirow[t]{2}{*}{ Variables } & \multicolumn{2}{|c|}{ With ATS/IDSA criteria } & \multicolumn{2}{|c|}{ With PSI } \\
\hline & $\begin{array}{c}\text { OR } \\
(95 \% \mathrm{Cl})\end{array}$ & $\mathrm{p}$-value & $\begin{array}{c}\text { OR } \\
(95 \% \mathrm{Cl})\end{array}$ & p-value \\
\hline \multicolumn{5}{|l|}{ Serotypes } \\
\hline $\mathrm{H}$ & 1 & & 1 & \\
\hline $\mathrm{O}$ & $\begin{array}{c}1.72 \\
(0.40-7.41)\end{array}$ & 0.46 & $\begin{array}{c}1.28 \\
(0.29-5.60)\end{array}$ & 0.73 \\
\hline L & $\begin{array}{c}7.02 \\
(1.72-28.61)\end{array}$ & $<0.01$ & $\begin{array}{c}5.28 \\
(1.29-21.58)\end{array}$ & $<0.05$ \\
\hline ATS/IDSA criteria & $\begin{array}{c}4.80 \\
(1.89-12.13)\end{array}$ & $<0.01$ & & \\
\hline PSI class $V$ & & & $\begin{array}{c}9.50 \\
(3.06-29.46)\end{array}$ & $<0.001$ \\
\hline Alcohol abuse & $\begin{array}{c}3.99 \\
(1.39-11.39)\end{array}$ & 0.01 & $\begin{array}{c}3.16 \\
(1.18-8.46)\end{array}$ & $<0.05$ \\
\hline Charlson score & $\begin{array}{c}1.30 \\
(1.08-1.57)\end{array}$ & $<0.01$ & $\begin{array}{c}1.22 \\
(1.01-1.46)\end{array}$ & $<0.05$ \\
\hline Age & $\begin{array}{c}1.02 \\
(0.99-1.05)\end{array}$ & 0.11 & & \\
\hline
\end{tabular}

H serotypes: 1, 5 and 7 F. L serotypes: 3, 6A, 6B, 8, 19F and 23F. O serotypes; non-L, non-H serotypes. ATS: American Thoracic Society; IDSA: Infectious Diseases Society of Americal; PSI: Pneumonia Severity Index.

that infection by L serotypes influenced mortality by itself, irrespective of the source of infection.

Conversely, in a multicentre study involving 760 patients with pneumococcal bacteraemia and after adjusting for baseline conditions and severity of illness, AlANEE et al. [13] did not find an independent association between mortality and specific serotypes or groups of serotypes (paediatric, conjugated and invasive serotypes), suggesting that host factors were more important than serotypes in determining severity and outcome.

In our study, serotypes 1, 5 and 7F were associated with lower severity according to PSI score (only $18 \%$ of patients showed PSI class V). Similar severity patterns have been reported in a study of patients with meningitis [23]. These serotypes were associated with an increased risk of complicated parapneumonic effusion or empyema, although serotype 1 accounts for $50 \%$ of episodes. Some authors have reported this association in paediatric populations [24], but not in adults [13].

Vaccination strategy is a key point to improve outcome in pneumococcal pneumonia. Unfortunately, the rate of PPV-23 vaccination in our cohort was low. Despite the effectiveness of PPV-23 vaccine in reducing the severity of the infection, some vulnerable cohorts remain: for instance, COPD patients and the elderly, who respond poorly to active immunisation with PPV23 and who might be a potential target population for PCV vaccines [25]. Our data suggest that the inclusion of some $L$ serotypes (mainly serotype 3 ) in PCV vaccines would improve survival in patients with high degrees of comorbidity.

Our study has several limitations. First, the great variability of isolated serotypes was a serious handicap in determining whether individual serotypes might have different degrees of influence on mortality or severity of illness. Secondly, the distribution of serotypes may differ in other geographic areas, especially with higher levels of immunisation with PPV-23. Thirdly, our findings may not be applicable to hospitalised patients without bacteraemia or to the paediatric population, in which different serotypes (mainly 7F) seem to be associated with excess-of-mortality [26]. Finally, the invasiveness or virulence of pneumococci may be different even among strains with the same serotype, suggesting that these properties are also associated with the genotype of the isolates. Recent studies demonstrated that multi-invasive locus sequence typing and comparative genomic hybridisation are promising techniques to distinguish strains that causes invasive disease from carrier strains [27].

In summary, our findings suggest that there is a group of serotypes associated with severe bacteraemic pneumococcal pneumonia in adults in Spain. These serotypes were independently associated with death; therefore, they should be included in new vaccines. Our data suggest that the global impact of the newer 13-valent vaccine in reducing mortality would be high in elderly population.

\section{SUPPORT STATEMENT}

This work was supported in part by: CIBERes 06/06/36, AGAUR 09/ SGR930, FISS 04/1500 and AI 07/90031.

\section{STATEMENT OF INTEREST}

None declared.

\section{ACKNOWLEDGEMENTS}

The authors would like to thank D. Suárez: (Fundació Parc Taulí, Sabadell, Spain) for statistical assessment and M. Maudsley (Language Service, University of Barcelona) for revision of the English version of the manuscript.

\section{REFERENCES}

1 Mandell LA, Wunderink RG, Anzueto A, et al. Infectious Diseases Society of America/American Thoracic Society consensus guidelines on the management of community-acquired pneumonia in adults. Clin Infect Dis 2007; 44: S27-S72.

2 Kalin M, Örtqvist A, Almela M, et al. Prospective study of prognostic factors in community-acquired bacteremic pneumococcal disease in 5 countries. J Infect Dis 2000; 182: 840-847.

3 Waterer GW, Quasney MW, Cantor RM, et al. Septic shock and respiratory failure in community-acquired pneumonia have different TNF polymorphism associations. Am J Respir Crit Care Med 2001; 163: 1599-1604.

4 Meehan TP, Fine MJ, Krumholz HM, et al. Quality of care, process, and outcomes in elderly patients with pneumonia. JAMA 1997; 278: 2080-2084.

5 Lujan M, Gallego M, Fontanals D, et al. Prospective observational study of bacteremic pneumococcal pneumonia: effect of discordant therapy on mortality. Crit Care Med 2004; 32: 625-631.

6 Baddour LM, Yu VL, Klugman KP, et al. Combination antibiotic therapy lowers mortality among severely ill patients with pneumococcal bacteremia. Am J Respir Crit Care Med 2004; 170: 440-444.

7 Aspa J, Rajas O, Rodriguez de Castro F, et al. Drug-resistant pneumococcal pneumonia: clinical relevance and related factors. Clin Infect Dis 2004; 38: 787-798.

8 Feikin DR, Schuchat A, Kolczak M, et al. Mortality from invasive pneumococcal pneumonia in the era of antibiotic resistance, 19951997. Am J Public Health 2000; 90: 223-229. 
9 Rello J, Lisboa T, Lujan M, et al. Severity of pneumococcal pneumonia associated with genomic bacterial load. Chest. 2009; 136: 832-840.

10 Henrichsen J. Six newly recognized types of Streptococcus pneumoniae. J Clin Microbiol 1995; 33: 2759-2762.

11 Brueggeman AB, Griffiths DT, Meats E, et al. Clonal relationships between invasive and carriage Streptococcus pneumoniae and serotype and clone-specific differences in invasive potential J Infect Dis 2003; 187: 1424-1432.

12 Sjöström K, Spindler C, Ortqvist A, et al. Clonal and capsular types decide whether pneumococci will act as a primary or opportunistic pathogen. Clin Infect Dis 2006; 42: 451-459.

13 Alanee SR, McGee L, Jackson D, et al. Association of serotypes of Streptococcus pneumoniae with disease severity and outcome in adults: an international study. Clin Infect Dis 2007; 45: 46-51.

14 Charlson ME, Pompei P, Ales KL, et al. A new method of classifying prognostic comorbidity in longitudinal studies: development and validation. J Chron Dis 1987; 40: 373-383.

15 Fine MJ, Auble TE, Yealy DM, et al. A prediction rule to identify low-risk patients with community-acquired pneumonia. $N$ Engl J Med 1997; 336: 243-250.

16 Sahn SS. Diagnosis and management of parapneumonic effusions and empyema. Clin Infect Dis 2007; 45: 1480-1486.

17 National Committee for Clinical Laboratory Standards. Performance standards for antimicrobial susceptibility testing. Seventeenth informational supplement. Document M100-S18. Wayne, National Committee for Clinical Laboratory Standards, 2008.

18 Vittinghoff E, McCulloch CE. Relaxing the rule of ten events per variable in logistic and Cox regression. Am J Epidemiol 2007; 165: 710-718.
19 Henriques B, Kalin M, Ortqvist A, et al. Molecular epidemiology of Streptococcus pneumoniae causing invasive disease in 5 countries. J Infect Dis 2000; 182: 833-839.

20 Martens P, Worm SW, Lundgren B, et al. Serotype-specific mortality from invasive Streptococcus pneumoniae disease revisited. BMC Infect Dis 2004; 4: 21-28.

21 Harboe ZB, Thomsen RW, Riis A. Pneumococcal serotypes and mortality following invasive pneumococcal disease: a populationbased cohort study. PLoS Med 2009; 6: e1000081.

22 Janssen A, Rodenburg G, van der Ende A. Invasive pneumococcal disease among adults: association among serotypes, disease characteristics and outcome. Clin Infect Dis 2009; 49: e23-e29.

23 Ostergaard C, Brandt C, Konradsen $\mathrm{HB}$, et al. Differences in survival, brain damage and cerebrospinal fluid cytokine kinetics due to meningitis caused by 3 different Streptococcus pneumoniae serotypes: evaluation in humans and in 2 experimental models. J Infect Dis 2004; 190: 1212-1220.

24 Obando I, Muñoz-Almagro C, Arroyo LA, et al. Pediatric parapneumonic empyema, Spain. Emerg Infect Dis 2008; 14: 1390-1397.

25 Dransfield MT, Nahm MH, Han MK, et al. Superior immune response to protein-conjugate versus free pneumococcal polysaccharide vaccine in chronic obstructive pulmonary disease. Am J Respir Crit Care Med 2009; 180: 499-505.

26 Rückinger S, von Kries R, Siedler A, et al. Association of serotype of Streptococcus pneumoniae with risk of severe and fatal outcome. Pediatr Infect Dis J 2009; 28: 118-122.

27 Obert C, Gao G, Sublett J, et al. Assessment of molecular typing methods to determine invasiveness and to differentiate clones of Streptococcus pneumoniae. Infect Genet Evol 2007; 7: 708-716. 